Keywords: ${ }^{18} \mathrm{~F}$-FDG-PET/CT; breast cancer; HER2; neoadjuvant chemotherapy; metabolic response; pathologic complete response

\title{
HER2-overexpressing breast cancer: FDG uptake after two cycles of chemotherapy predicts the outcome of neoadjuvant treatment
}

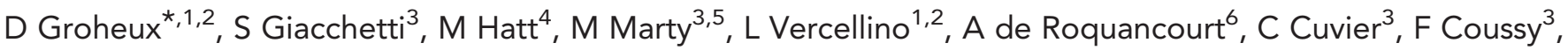
M Espié ${ }^{3}$ and E Hindié ${ }^{7}$

${ }^{1}$ Nuclear Medicine, Saint-Louis Hospital, 1 avenue Claude Vellefaux, Paris 75475, France; ${ }^{2}$ B2T, Doctoral School, IUH, University of Paris VII, Paris, France; ${ }^{3}$ Breast Diseases Unit, Saint-Louis Hospital, Paris, France; ${ }^{4}$ INSERM, UMR 1101 LaTIM, Brest, France; ${ }^{5}$ Centre for Therapeutic Innovation, Saint-Louis Hospital, Paris, France; ${ }^{6}$ Pathology, Saint-Louis Hospital, Paris, France and ${ }^{7}$ Nuclear Medicine, Bordeaux University Hospital, University of Bordeaux, Bordeaux, France

Background: Pathologic complete response ( $\mathrm{pCR}$ ) to neoadjuvant treatment (NAT) is associated with improved survival of patients with HER2 + breast cancer. We investigated the ability of interim positron emission tomography (PET) regarding early prediction of pathology outcomes.

Methods: During 61 months, consecutive patients with locally advanced or large HER2 + breast cancer patients without distant metastases were included. All patients received NAT with four cycles of epirubicin + cyclophosphamide, followed by four cycles of docetaxel + trastuzumab. ${ }^{18} \mathrm{~F}$-fluorodeoxyglucose $\left({ }^{18} \mathrm{~F}-\mathrm{FDG}\right)-\mathrm{PET} / \mathrm{computed}$ tomography (CT) was performed at baseline $\left(P E T_{1}\right)$ and after two cycles of chemotherapy $\left(P E T_{2}\right)$. Maximum standardised uptake values were measured in the primary tumour as well as in the axillary lymph nodes. The correlation between pathologic response and SUV parameters (SUV $\max$ at PET , $\mathrm{PET}_{2}$ and $\Delta S U V_{\max }$ ) was examined with the $t$-test. The predictive performance regarding the identification of non-responders was evaluated using receiver operating characteristics $(\mathrm{ROC})$ analysis.

Results: Thirty women were prospectively included and 60 PET/CT examination performed. At baseline, 22 patients had PET + axilla and in nine of them ${ }^{18} \mathrm{~F}$-FDG uptake was higher than in the primary tumour. At surgery, 14 patients (47\%) showed residual tumour (non-pCR), whereas $16(53 \%)$ reached $\mathrm{PCR}$. Best prediction was obtained when considering the absolute residual $S U V_{\max }$ value at $\mathrm{PET}_{2}\left(A U C=0.91\right.$ ) vs 0.67 for $S U V_{\max }$ at $P E T_{1}$ and 0.86 for $\triangle S U V_{\max }$. The risk of non-pCR was $92.3 \%$ in patients with any site of residual uptake $>3$ at $\mathrm{PET}_{2}$, no matter whether in breast or axilla, vs $11.8 \%$ in patients with uptake $\leqslant 3$ $(P=0.0001)$. The sensitivity, specificity, PPV, NPV and overall accuracy of this cutoff were, respectively: $85.7 \%, 93.8 \%, 92.3 \%$, $88.2 \%$ and $90 \%$.

Conclusion: The level of residual ${ }^{18}$ F-FDG uptake after two cycles of chemotherapy predicts residual disease at completion of NAT with chemotherapy + trastuzumab with high accuracy. Because many innovative therapeutic strategies are now available (e.g., addition of a second HER2-directed therapy or an antiangiogenic), early prediction of poor response is critical.

*Correspondence: Dr D Groheux; E-mail: dgroheux@yahoo.fr

Received 18 May 2013; revised 17 July 2013; accepted 21 July 2013; published online 13 August 2013

(c) 2013 Cancer Research UK. All rights reserved 0007-0920/13 
Neoadjuvant chemotherapy was initially developed for primary inoperable breast cancer, and is now also widely used in operable but large breast cancer not eligible to breast-conserving therapy (NCCN Guidelines, 2013). Positron emission tomography/computed tomography with ${ }^{18} \mathrm{~F}$-fluorodeoxyglucose $\left({ }^{18} \mathrm{~F}\right.$-FDG-PET/CT) is a useful staging modality in these patients (Fuster et al, 2008; Groheux et al, 2012a, 2013a). Moreover, some studies have demonstrated a correlation between early changes in ${ }^{18} \mathrm{~F}-\mathrm{FDG}$ primary tumour uptake after one or two courses of chemotherapy and the extent of pathology response at completion of treatment, at the tumour level (Schwarz-Dose et al, 2009; Wang et al, 2012), as well as in axillary lymph nodes (Straver et al, 2010; Rousseau et al, 2011). However, the ability to implement ${ }^{18} \mathrm{~F}$-FDG-PET/CT as a surrogate marker for treatment efficacy remains unclear because of substantial heterogeneities across studies and also because breast cancer cannot be examined as a single entity (Groheux et al, 2011a, 2012b, 2013b; Humbert et al, 2012). Breast cancer comprises different phenotypes with different response rates to chemotherapy, different treatment options and different prognoses (NCCN Guidelines, 2013). We therefore suggested that the clinical aims of early ${ }^{18} \mathrm{~F}$-FDG monitoring and the criteria used to predict efficacy should be determined in specific subgroups (Groheux et al, 2011a, 2012b, 2013b).

Overexpression of the HER2 receptor occurs in roughly $20 \%$ of breast tumours. The prognosis of this aggressive entity has been improved with the advent of trastuzumab therapy, an antibody targeting the HER2 receptor (Gianni et al, 2010). In the neoadjuvant setting, pathologic complete response (pCR) at surgery is higher with the addition of trastuzumab and is correlated with improved outcomes, suggesting that it may serve as a surrogate marker of clinical benefit (Gianni et al, 2010; Untch et al, 2011; Von Minckwitz et al, 2012). Identifying poor responders before completion of neoadjuvant treatment (NAT) might be useful for improving outcome by allowing an early switch to a different chemotherapy regimen, and/or the use of more than one targeted therapy (Baselga et al, 2012; Gianni et al, 2012; Guarneri et al, 2012; Pierga et al, 2012). The objective of this prospective study was to assess the value of interim ${ }^{18} \mathrm{~F}$-FDG-PET/CT for early identification of HER2 + breast cancer patients who will not achieve pCR with a conventional chemotherapy/trastuzumab NAT.

\section{MATERIALS AND METHODS}

Patients. During 61 months, patients with clinical stage II or III HER2 + breast cancer seen at the breast disease unit of Saint Louis hospital, and scheduled for NAT were included. All patients underwent an ${ }^{18} \mathrm{~F}$-FDG-PET/CT scan at baseline $\left(\mathrm{PET}_{1}\right)$ and another scan after two cycles of chemotherapy $\left(\mathrm{PET}_{2}\right)$. Patients with distant metastases identified at initial staging were not included, because these patients receive treatments tailored to metastatic state and patient characteristics. After completion of NAT, all patients underwent surgery and response to treatment was assessed by pathology examination of surgical specimens. The study followed the guidelines of the institutional ethical committee with informed patient consent.

Neoadjuvant treatment. All patients received four cycles of epirubicin $\left(75 \mathrm{mg} \mathrm{m}^{-2}\right)$ plus cyclophosphamide $\left(750 \mathrm{mg} \mathrm{m}^{-2}\right)$ administered every 3 weeks, followed by four courses of docetaxel $\left(100 \mathrm{mg} \mathrm{m}^{-2}\right.$ every 3 weeks) plus trastuzumab $\left(8 \mathrm{mg} \mathrm{kg}^{-1}\right.$ loading dose, followed by $6 \mathrm{mg} \mathrm{kg}^{-1}$ every 3 weeks).

${ }^{18}$ F-FDG-PET/CT imaging. Patients fasted for $6 \mathrm{~h}$. Blood glucose level had to be $<7 \mathrm{mmoll}^{-1}$. ${ }^{18} \mathrm{~F}$-fluorodeoxyglucose $\left(5 \mathrm{MBq} \mathrm{kg}^{-1}\right.$; not exceeding $500 \mathrm{MBq}$ ) was administered $60 \mathrm{~min}$ before data were acquired on a Gemini XL PET/CT scanner (Philips Healthcare, Eindhoven, The Netherlands). Computed tomography was acquired first $(120 \mathrm{kV}$; 100mAs; no contrast enhancement). Positron emission

tomography three-dimensional (3D) data were acquired in a listmode format with 2 min per bed position, and images were reconstructed using a 3D row-action maximum-likelihood algorithm.

Positron emission tomography/CT images were interpreted by two nuclear medicine specialists blinded to the patient's record. ${ }^{18}$ F-fluorodeoxyglucose uptake was expressed as SUV (standardised uptake value). A 3D region of interest (3D-ROI) was drawn around the primary tumour and around axillary lymph nodes when present. Maximum SUV value within each ROI was recorded and used for the study analysis. The lesion with the highest initial uptake (either the primary tumour or an axillary lymph node) was considered the main 'target' to assess. The change in $\mathrm{SUV}_{\max }$ after two cycles of chemotherapy was expressed as follows:

$$
\Delta \mathrm{SUV}_{\max }(\%)=100 \times\left(\mathrm{PET}_{2} \cdot \mathrm{SUV}_{\max }-\mathrm{PET}_{1} \cdot \mathrm{SUV}_{\max }\right) / \mathrm{PET}_{1} \cdot \mathrm{SUV}_{\max } .
$$

Tumour histology and immunohistochemistry analysis. Breast cancer diagnosis was performed on a core-needle biopsy. Tumour grade was determined using the modified Scarff-Bloom-Richardson classification. Tumours were classified as oestrogen receptor positive $(\mathrm{ER}+)$ if showing moderate or high positivity ( 2 or $3+)$ of at least $10 \%$ of cells. The same criteria were used for progesterone receptor (PR) status. Tumours were classified as

Table 1. Main tumour characteristics in the 30 patients No. of patients (\%)

AJCC clinical stage ${ }^{a}$

\begin{tabular}{l|l}
\hline IIA & $5(16)$ \\
IIB & $9(30)$ \\
IIIA & $8(27)$ \\
IIIB & $6(20)$ \\
IIIC & $2(7)$
\end{tabular}

\section{Tumour type}

Invasive ductal

Metaplastic

Lobular

29 (97)

1 (3)

0

\section{Grade}

Grade 1

Grade 2

Grade 3

0

9 (30)

$21(70)$

Oestrogen receptor status

\begin{tabular}{|l|r|}
\hline Positive & $12(40)$ \\
Negative & $18(60)$
\end{tabular}

Progesterone receptor status

Positive $3(10)$

Negative 27 (90)

\section{Surgery}

Breast-conserving surgery

Mastectomy

$14(47)$

$16(53)$

\section{Pathologic response}

\begin{tabular}{|l|r}
\hline $\mathrm{PCR}$ & $16(53)$ \\
\hline
\end{tabular}

Non-pCR

$14(47)$

Abbreviations: $\mathrm{AJCC}=$ American Joint Committee on Cancer; $\mathrm{PCR}=$ pathologic complete response.

${ }^{a}$ Clinical stage according to clinical examination and conventional imaging findings; AJCC version 7 . 
HER2 overexpression (HER2 +) if IHC staining results were $3+$ (uniform, intense membrane staining of $>30 \%$ of invasive tumour cells). Equivocal results $(2+)$ were further tested by FISH or SISH (Wolff et al, 2007).

Pathology assessment at completion of NAT. Pathologic complete response (pCR) was defined as no evidence of residual invasive cancer, both in breast tissue and lymph nodes. Absence of in situ carcinoma was not required to define pCR.

Statistical analysis. Statistical analyses were performed using Medcalc (MedCalc Software, Ostend, Belgium). $P<0.05$ was considered statistically significant. Maximum standardised uptake values were measured in the primary tumour as well as in the axillary lymph nodes. The correlation between pathologic response and SUV parameters $\left(\mathrm{SUV}_{\max }\right.$ at baseline, at two cycles and
$\left.\Delta \mathrm{SUV}_{\max }\right)$ was examined with the $t$-test and log-transformed data because of non-normality of the distributions. The predictive performance regarding the identification of non-responders was evaluated using ROC analysis. Correlations between clinical or biological parameters (axillary status, tumour grade, ER expression) and pathologic response were assessed with the $t$-test. The influence of biological parameters on $\mathrm{SUV}_{\max }$ value of primary tumour on baseline PET study was also assessed with the $t$-test.

\section{RESULTS}

Tumours characteristics and pathological response. Thirty consecutive patients with large or locally advanced HER $2+$ breast

Table 2. PET findings and pathologic response for 30 patients with HER2 + breast cancer

$$
\mathrm{PET}_{1} \mathrm{SUV}_{\max } \quad \mathrm{PET}_{2} \mathrm{SUV}_{\max }
$$

\begin{tabular}{|c|c|c|c|c|c|c|c|c|c|}
\hline Patient no. & Stage ${ }^{a}$ & $\begin{array}{l}\text { Primary } \\
\text { tumour }\end{array}$ & $\begin{array}{l}\text { Lymph } \\
\text { nodes }\end{array}$ & Target & $\begin{array}{l}\text { Primary } \\
\text { tumour }\end{array}$ & $\begin{array}{l}\text { Lymph } \\
\text { nodes }\end{array}$ & $\begin{array}{c}\Delta S U V_{\text {max }} \text { primary } \\
\text { tumour }\end{array}$ & $\begin{array}{c}\Delta \text { SUV }_{\max } \\
\text { target }\end{array}$ & $\begin{array}{c}\text { Pathology } \\
\text { response }\end{array}$ \\
\hline 1 & IIIB & 4.6 & 23.9 & $N$ & 1.1 & 0.9 & $-76 \%$ & $-96 \%$ & $\mathrm{pCR}$ \\
\hline 2 & IIIA & 2.7 & 12.1 & $N$ & 1.9 & 1.5 & $-30 \%$ & $-88 \%$ & $\mathrm{pCR}$ \\
\hline 3 & IIB & 9.1 & 5.9 & $T$ & 2.4 & 0.8 & $-74 \%$ & $-74 \%$ & $\mathrm{pCR}$ \\
\hline 4 & IIIB & 7.9 & 8.7 & $\mathrm{~N}$ & 2.4 & 0.8 & $-70 \%$ & $-91 \%$ & $\mathrm{pCR}$ \\
\hline 5 & IIIB & 5.5 & 8.3 & $N$ & 2.2 & 1.8 & $-60 \%$ & $-78 \%$ & $\mathrm{pCR}$ \\
\hline 6 & $\mathrm{IIB}$ & 7.6 & - & $T$ & 2.3 & - & $-70 \%$ & $-70 \%$ & $\mathrm{pCR}$ \\
\hline 7 & IIIA & 7.1 & 6.2 & $\mathrm{~T}$ & 2.4 & 1.1 & $-66 \%$ & $-66 \%$ & $\mathrm{pCR}$ \\
\hline 8 & IIIB & 7.1 & 2 & $T$ & 1.8 & 0.7 & $-75 \%$ & $-75 \%$ & $\mathrm{pCR}$ \\
\hline 9 & IIIA & 7 & 1.9 & $\mathrm{~T}$ & 2.6 & 1.2 & $-63 \%$ & $-63 \%$ & $\mathrm{pCR}$ \\
\hline 10 & $\mathrm{IIB}$ & 6.2 & 6.7 & $N$ & 1.3 & 0.9 & $-79 \%$ & $-87 \%$ & $\mathrm{pCR}$ \\
\hline 11 & IIIB & 6.1 & - & $T$ & 2.3 & - & $-62 \%$ & $-62 \%$ & $\mathrm{pCR}$ \\
\hline 12 & IIIA & 5.7 & 3 & $T$ & 3.6 & 1.2 & $-37 \%$ & $-37 \%$ & $\mathrm{pCR}$ \\
\hline 13 & IIIA & 5.2 & 4.6 & $\mathrm{~T}$ & 1.2 & 0.8 & $-77 \%$ & $-77 \%$ & $\mathrm{pCR}$ \\
\hline 14 & $\| \mathrm{A}$ & 4.7 & - & $T$ & 2.2 & - & $-53 \%$ & $-53 \%$ & $\mathrm{pCR}$ \\
\hline 15 & IIIB & 2.6 & 4.4 & $N$ & 2.4 & 2.9 & $-41 \%$ & $-34 \%$ & $\mathrm{pCR}$ \\
\hline 16 & IIIA & 2.4 & 2.2 & $T$ & 1.5 & 0.6 & $-38 \%$ & $-38 \%$ & $\mathrm{pCR}$ \\
\hline 17 & IIIC & 18.2 & 19.5 & $N$ & 14.1 & 24.1 & $-23 \%$ & $+24 \%$ & non-pCR \\
\hline 18 & $\| \mathrm{A}$ & 18.8 & - & $T$ & 12.4 & - & $-34 \%$ & $-34 \%$ & non-pCR \\
\hline 19 & IIIB & 17 & 12.3 & $\mathrm{~T}$ & 6.3 & 1.5 & $-63 \%$ & $-63 \%$ & non-pCR \\
\hline 20 & IIIA & 11 & 16.2 & $N$ & 5.9 & 13.4 & $-46 \%$ & $-17 \%$ & non-pCR \\
\hline 21 & IIB & 15.5 & 5.6 & $T$ & 5.9 & 1.1 & $-62 \%$ & $-62 \%$ & non-pCR \\
\hline 22 & IIIC & 12.1 & 11.9 & $\mathrm{~T}$ & 5.4 & 2.6 & $-55 \%$ & $-55 \%$ & non-pCR \\
\hline 23 & IIB & 11.5 & 7.5 & $T$ & 10 & 5.5 & $-13 \%$ & $-13 \%$ & non-pCR \\
\hline 24 & IIIB & 10.1 & - & $\mathrm{T}$ & 3.3 & - & $-67 \%$ & $-67 \%$ & non-pCR \\
\hline 25 & IIB & 3.8 & 7 & $N$ & 3.5 & 7.2 & $-8 \%$ & $+3 \%$ & non-pCR \\
\hline 26 & IIIB & 6.9 & 3.2 & $T$ & 3.4 & 0.9 & $-51 \%$ & $-51 \%$ & non-pCR \\
\hline 27 & IIIA & 6.7 & 6.6 & $T$ & 5.8 & 3.7 & $-13 \%$ & $-13 \%$ & non-pCR \\
\hline 28 & $\| \mathrm{A}$ & 6.2 & - & $\mathrm{T}$ & 6.3 & - & $+2 \%$ & $+2 \%$ & non-pCR \\
\hline 29 & IIA & 5.8 & - & $T$ & 2.4 & - & $-59 \%$ & $-59 \%$ & non-pCR \\
\hline 30 & $\| \mathrm{A}$ & 3.2 & - & $T$ & 1.4 & - & $-56 \%$ & $-56 \%$ & non-pCR \\
\hline \multicolumn{10}{|c|}{ 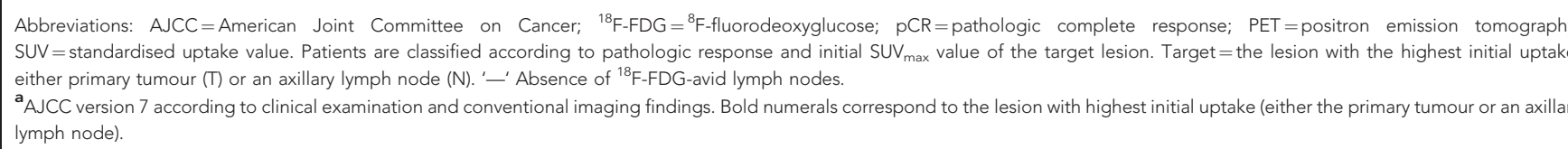 } \\
\hline
\end{tabular}


cancer and no distant metastases at baseline staging were included (Table 1). Eight patients could not be included because distant metastases were discovered on baseline ${ }^{18}$ F-FDG imaging.

All patients received the eight courses of NAT. At completion of NAT, 14 patients received breast-conserving surgery and the others mastectomy. All patients had axillary lymph node dissection. Histopathology showed residual invasive disease (non-pCR) in 14 (47\%), whereas 16 patients (53\%) had pCR with no residual invasive disease in axilla and in breast (in situ carcinoma was present in three of them).

Absolute value of SUV max at PET1 and PET2. Individual PET data and pathological response are outlined in Table 2 and associations between PET parameters with response at completion of chemotherapy are shown in Table 3.

At $\mathrm{PET}_{1}, \mathrm{SUV}_{\max }$ of breast tumour ranged between 2.4 and 18.8 $($ mean $=7.9)$. Twenty-two patients had PET + axilla $\left(\mathrm{SUV}_{\max }\right.$ : 1.9-23.9; mean $=8.2$ ), and in nine of them ${ }^{18}$ F-FDG uptake was higher than in the primary tumour.

Maximum standardised uptake value of the site with highest uptake (target lesion) at $\mathrm{PET}_{1}$ ranged between 2.4 and 23.9 (mean $=9.4)$. There was some, nonsignificant, correlation between high baseline $\mathrm{SUV}_{\max }$ of the target lesion and residual disease at completion of NAT $(P=0.08)$ (Figure 1$)$.

At $\mathrm{PET}_{2}$, breast tumour $\mathrm{SUV}_{\max }$ ranged between 1.1 and 14.1 $($ mean $=4.0)$ and $\mathrm{SUV}_{\max }$ in lymph nodes ranged between 0.6 and $24.1($ mean $=3.4)$.

There was a strong correlation between the highest residual ${ }^{18}$ F-FDG uptake at $\mathrm{PET}_{2}$ and pathological response (mean residual $\mathrm{SUV}_{\max }=7.7$ in non-pCR $v s 2.1$ in pCR patients, $P=0.0001$; Table 3). Best prediction was obtained with a cutoff of $S_{U} V_{\max }=3$ (Figure 1), which allowed identification of poor responders (nonpCR) with a sensitivity, specificity, PPV, NPV and overall accuracy of, respectively: $85.7 \%$ (12 out of 14 ), $93.8 \%$ (15 out of 16 ), $92.3 \%$ (12 out of 13 ), $88.2 \%$ (15 out of 17 ) and $90 \%$ (27 out of 30 ).

The risk of non-pCR was $92.3 \%$ in patients with any site of residual uptake $>3$ on interim PET, no matter whether in breast or axilla, vs $11.8 \%$ in patients with uptake $\leqslant 3(P=0.0001)$.

The accuracy of $\mathrm{PET}_{2} \mathrm{SUV}_{\max }$ was even higher $(95.5 \% ; 21$ out of 22) in the subset of patients with positive nodes on baseline PET (i.e., those with more than one ${ }^{18} \mathrm{~F}-\mathrm{FDG}$ uptake site).

Evolution of SUV max between PET1 and PET2. The mean $\Delta S U_{\text {max }}$ for the target lesion (site of highest initial uptake, either breast primary or axillary node) was $-51 \%$ (range: -95 to $+24 \%$ )
(Table 3). The mean decrease in $\mathrm{SUV}_{\max }$ was significantly larger in patients who achieved pCR $(-66 \pm 18 \%)$ than in those who did not $(-33 \pm 30 \%)(P=0.001)$ (Figure 1$)$. Using a cutoff of $<62 \%$ decrease in $\mathrm{SUV}_{\max }$, residual disease (non-pCR) was depicted with a sensitivity of $85.7 \%$, a specificity of $75 \%$, and an overall accuracy of $80 \%$ ( 24 out of 30 ).

Prediction based on $\Delta \mathrm{SUV}_{\max }$ was more modest when considering only the primary tumour. In this case, the cutoff of $62 \%$ decrease in $\mathrm{SUV}_{\text {max }}$ predicted pathology outcomes with an overall accuracy of $73.3 \%$ (22 out of 30 ) (sensitivity $85.7 \%$, specificity $62.5 \%)$.

Optimal PET parameter for prediction (ROC analysis). Figure 2 shows the AUC for prediction of pathology outcomes for each of the following PET parameters: $S_{U} V_{\max }$ value of target lesion at $\mathrm{PET}_{1} ; \mathrm{SUV}_{\max }$ value of target lesion at $\mathrm{PET}_{2}$; and $\Delta \mathrm{SUV}_{\max }$ of the target lesion. Absolute $\mathrm{SUV}_{\max }$ at $\mathrm{PET}_{2}$ offers the highest performance (AUC $=0.91$ vs 0.67 for $\mathrm{SUV}_{\max }$ at $\mathrm{PET}_{1}$ and 0.86 for $\left.\Delta S U V_{\text {max }}\right)$. This is also the case when examining the subset of 22 patients with initially node-positive axilla $(\mathrm{AUC}=0.99$ vs 0.73 for $\mathrm{SUV}_{\max }$ at $\mathrm{PET}_{1}$ and 0.89 for $\triangle \mathrm{SUV}_{\max }$ ).

Figures 3 and 4 show initial and interim PET/CT images in two patients, a patient who achieved pCR (Figure 3 ) and one who did not (Figure 4).

Impact of clinical and biological parameters. Baseline $S U V_{\max }$ values were significantly higher in grade 3 than in grade 2 primary breast tumours (mean: $9.0 \pm 4.8$ vs $5.5 \pm 2.7 ; P<0.03$ ), corroborating our previous findings in a general population of breast cancer (Groheux et al, 2011b). However, in these patients with HER2 overexpression, there was no significant difference in baseline $\mathrm{SUV}_{\max }$ values between $\mathrm{ER}+$ and $\mathrm{ER}$ - tumours (mean: $8.3 \pm 4.9$ vs $7.7 \pm 4.4 ; P=0.7)$.

Neither initial axillary status $(\mathrm{N}+v s \mathrm{~N} 0)(P=0.3)$ nor $\mathrm{ER}$ status $(P=0.8)$, or tumour grade $(P=0.5)$, showed statistically significant association with pathological response.

\section{DISCUSSION}

In the present series of patients with large or locally advanced HER2 + non-metastatic breast cancer who received NAT with four cycles of epirubicin + cyclophosphamide, followed by four cycles of docetaxel + trastuzumab, pCR was achieved in $53 \%$ of patients, whereas $47 \%$ had residual tumour at surgery,

Table 3. Associations between PET parameters with response at completion of chemotherapy

\begin{tabular}{|c|c|c|c|c|}
\hline \multicolumn{4}{|c|}{ Mean \pm s.d. (median; min; max) } & \multirow[b]{2}{*}{$P$-value } \\
\hline PET parameter & All patients $(n=30)$ & Non-pCR $(n=16)$ & pCR $(n=14)$ & \\
\hline \multicolumn{5}{|l|}{ Primary tumour } \\
\hline $\mathrm{SUV}_{\max } \mathrm{PET}_{1}$ & $7.9 \pm 4.5(6.8 ; 2.4 ; 18.8)$ & $10.5 \pm 5.3(10.6 ; 3.2 ; 18.8)$ & $5.7 \pm 2.0(5.9 ; 2.4 ; 9.1)$ & 0.006 \\
\hline $\mathrm{SUV}_{\max } \mathrm{PET}_{2}$ & $4.0 \pm 3.3(2.4 ; 1.1 ; 14.1)$ & $6.2 \pm 3.7(5.9 ; 1.4 ; 14.1)$ & $2.1 \pm 0.6(2.3 ; 1.1 ; 3.6)$ & 0.0001 \\
\hline$\Delta S U V_{\max }$ & $-50 \pm 24(-57 ;-79 ;+2)$ & $-39 \pm 24(-49 ;-67 ;+2)$ & $-59 \pm 20(-65 ;-79 ;-8)$ & 0.01 \\
\hline \multicolumn{5}{|l|}{ Target } \\
\hline $\mathrm{SUV}_{\max } \mathrm{PET}_{1}$ & $9.4 \pm 5.3(7.1 ; 2.4 ; 23.9)$ & $11.2 \pm 5.4(10.8 ; 3.2 ; 19.5)$ & $7.9 \pm 4.8(7.1 ; 2.4 ; 23.9)$ & 0.08 \\
\hline $\mathrm{SUV}_{\max } \mathrm{PET}_{2}$ & $4.7 \pm 4.9(2.5 ; 1.1 ; 24.1)$ & $7.7 \pm 5.6(6.1 ; 1.4 ; 24.1)$ & $2.1 \pm 0.7(2.3 ; 1.1 ; 3.6)$ & 0.0001 \\
\hline$\Delta S U V_{\max }$ & $-51 \pm 29(-60 ;-95 ;+24)$ & $-33 \pm 30(-42 ;-67 ;+24)$ & $-66 \pm 18(-71 ;-95 ;-34)$ & 0.001 \\
\hline
\end{tabular}



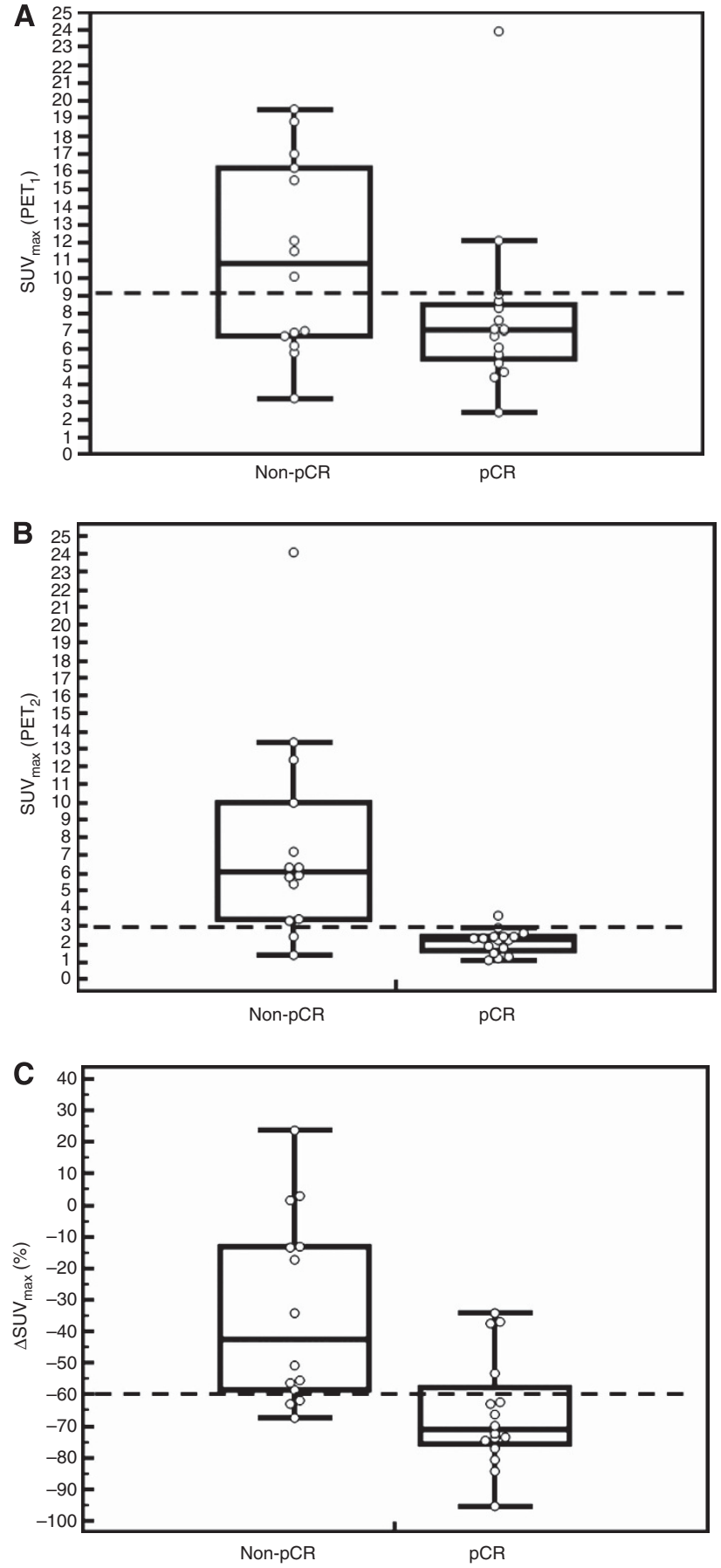

Figure 1. Distributions of absolute values of $\mathrm{SUV}_{\text {max }}$ at $\mathrm{PET}_{1}(\mathbf{A})$ and $\mathrm{PET}_{2}$ (B) of the site with highest uptake (breast primary cancer or axillary node) and $\triangle S U V_{\text {max }}$ values (C), for non-pCR and $\mathrm{pCR}$ patients; the optimal cutoff values of $\mathrm{SUV}_{\max }\left(\mathrm{PET}_{1}\right)=9.1, \mathrm{SUV}_{\max }\left(\mathrm{PET}_{2}\right)=3$ and $\Delta S U V_{\max }=-62 \%$ are drawn on the graphs (dashed lines).

which is in agreement with other reports (Untch et al, 2011; Humbert et al, 2012).

We examined the ability of ${ }^{18}$ F-FDG-PET/CT in predicting pathology outcomes after only two cycles of chemotherapy. The lesion with the baseline highest ${ }^{18} \mathrm{~F}$-FDG uptake was considered the target lesion for assessing the change in uptake under therapy $(\triangle \mathrm{SUV})$. Axilla was thus the target in nine patients in whom ${ }^{18}$ F-FDG uptake in axillary nodes was higher than in the primary tumour (Table 2). This refinement offered a modest improvement in PET prediction based on $\triangle \mathrm{SUV}$. Using $62 \%$ decrease in $\mathrm{SUV}_{\max }$ at two cycles as cutoff, pathology findings were predicted with an overall accuracy of $80 \%$ ( 24 out of 30 ), whereas analysis taking into consideration the primary tumour alone offered a lower accuracy of $73.3 \%$ ( 22 out of 30 ). This accuracy offered by $\Delta$ SUV was similar to that (76\%) reported by Humbert et al (2012). Koolen et al (2013) found lower predictive value of $\triangle$ SUV in HER2 + patients.

However, as evidenced in the present series, $\triangle \mathrm{SUV}$ is probably not the appropriate PET parameter for assessing response in this breast cancer phenotype. The absolute value of residual ${ }^{18} \mathrm{~F}-\mathrm{FDG}$ uptake on interim PET, whatever the site of residual uptake (breast or axilla), was the single most important parameter in the prediction of pathology outcome (Figures 1 and 2). Any site of residual ${ }^{18}$ F-FDG uptake with an $\mathrm{SUV}_{\max }>3$ was predictive of non-pCR with a sensitivity of $85.7 \%$, a specificity of $93.8 \%$ and an overall accuracy of $90 \%$ (27 out of 30 ). Accuracy was even higher (95.5\%) when considering the 22 patients who had ${ }^{18}$ F-FDG-avid node on baseline PET.

These findings are in contrast with those we obtained in patients with triple-negative phenotype, in whom $\triangle \mathrm{SUV}$ was the important determinant (Groheux et al, 2012b), further confirming our thought that each breast cancer phenotype should be examined as a separate entity when assessing the value of ${ }^{18} \mathrm{~F}$-FDG-PET for monitoring response to treatment (Groheux et al, 2011a).

Among PET parameters, other than $\mathrm{SUV}_{\max }$, metabolic tumour volume (MTV) and total lesion glycolysis (TLG) are potentially useful parameters. In a previous pilot series, we have compared the value of various PET image-derived indices in predicting response to therapy in locally advanced breast cancer. We found that TLG had better predictive value than $S_{U V} V_{\max }$ for the subgroup of luminal tumours $(\mathrm{RH}+/ \mathrm{HER} 2-)$, but not in triple-negative breast cancer or HER2 + breast cancer (Hatt et al, 2013). In the present study that deals with the HER2 + subtype we therefore did not include MTV or TLG parameters.

Any study investigating sequential PET scans needs to ensure the highest reproducibility possible regarding the acquisition protocol (Boellaard et al, 2010). In this study, imaging conditions were optimised to reduce the variability of the acquisition to a minimum.

Our findings show that the quality of early response to chemotherapy is an important determinant of the final outcome of a sequential treatment combining an anthracycline-based initial regimen (here epirubicin + cyclophosphamide), followed by trastuzumab + a taxane (here docetaxel). Such regimen is one of the more widely used (NCCN Guidelines, 2013). As stated in the National Comprehensive Cancer Network guidelines, 'Retrospective evidence suggests that anthracycline-based chemotherapy regimens may be superior to non-anthracycline-based regimens in patients with HER2 + tumours' (NCCN Guidelines, 2013). Trastuzumab is given sequentially and not concurrently with anthracyclines because of cardiac toxicity (NCCN Guidelines, 2013). Also, there is a synergistic activity of trastuzumab and docetaxel (Arnould et al, 2006).

There are many other neoadjuvant schemes in HER2 + patients. In a study of 37 patients treated from the outset with trastuzumab + docetaxel ( \pm carboplatin), the average $\Delta \mathrm{SUV}_{\max }$ after one course was $-71 \%$ in pCR $v s-47 \%$ in non-pCR cases $(P=0.01)$ (Humbert et al, 2012). A cutoff of $\Delta S U V_{\max }$ of $-75 \%$ offered an accuracy of $76 \%$ in predicting pathology outcome (Humbert et al, 2012). On the basis of these findings, the authors planned a randomised trial to assess the benefit of adding bevacizumab when the decrease in $\mathrm{SUV}_{\max }$ is $<70 \%$ after the first course of docetaxel + trastuzumab (Cochet et al, 2012). Prediction under anti-HER2 therapy alone is also possible, although the extent of decrease in $\mathrm{SUV}_{\max }$ might be more modest. In a report on a sequential schema where the authors introduced 

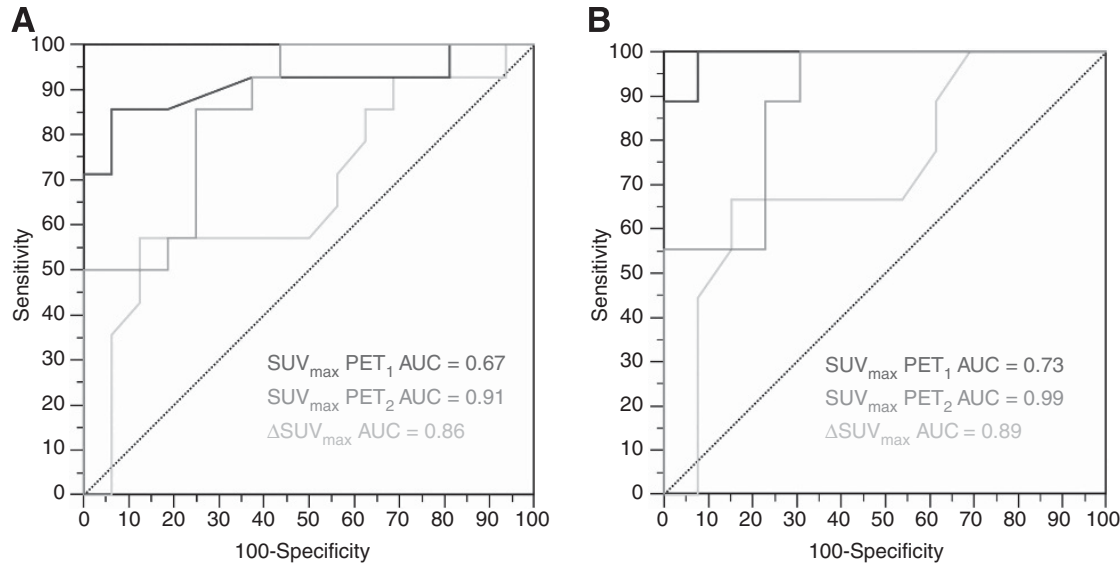

Figure 2. Areas under the receiver operator curves (AUCs) showing the ability of different PET parameters at predicting residual disease

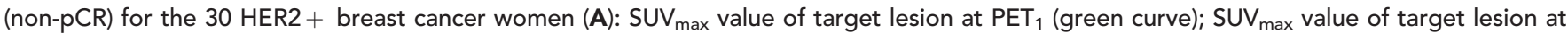
$\mathrm{PET}_{2}$ (blue curve); and $\Delta \mathrm{SUV}_{\max }$ of the target lesion (orange curve). The absolute $\mathrm{SUV}_{\max }$ value at $\mathrm{PET}_{2}$ provides the highest performance $(A \cup C=0.91$ ). Similar curves are drawn for the subset of 22 patients with baseline node-positive axilla (B). Again, best prediction is offered by the absolute $\mathrm{SUV}_{\max }$ value at $\mathrm{PET}_{2}(\mathrm{AUC}=0.99)$. The full colour version of this figure is available at British Journal of Cancer online.


Figure 3. A 48-year-old woman (patient no. 10; Table 2) with HER2-overexpressing tumour of left breast. Positron emission tomography and $\mathrm{PET} / \mathrm{CT}$ fusion images of the primary tumour and axillary lymph nodes, at baseline (A-D), and after two courses of chemotherapy (E-H). At baseline, SUV max was 6.2 in breast tumour (A and $\mathbf{B}$ ) and 6.7 in a lymph node (C and $\mathbf{D}$ ). At PET ${ }_{2}, \mathrm{SUV}_{\max }$ was 1.3 in breast tumour (arrow, $\mathbf{E}$ and $\mathbf{F}$ ) $(\Delta S U V=-79 \%)$ and 0.9 in the lymph node (arrow, $\mathbf{G}$ and $H)(\Delta S U V=-87 \%)$. At completion of NAT, breast and axillary surgery showed 'pCR'.

first anti-HER2-therapy alone for 6 weeks (either trastuzumab, or lapatinib, or their combination), $60 \%$ of patients showed a metabolic response (decrease in SUV > 25\%; EORTC criteria). The pCR rate was two times as high in PET 'responders' compared with 'non-responders' (44\% vs $19 \%, P=0.05$ ) (Baselga et al, 2012; Gebhart et al, 2012). In all these studies, assessing the efficacy of PET prediction after having introduced trastuzumab, the accuracy of interim PET was rather modest, and lower than in the present series (90\%). One explanation could be that the decrease in ${ }^{18} \mathrm{~F}$-FDG uptake under trastuzumab is not a pure reflect of cell killing but also reflects other specific impact on glucose metabolism, such as reduction in Glut1 and in Hexokinase II activity, as evidenced in animal studies with tumour xenografts (Smith et al, 2013). False positives might also result from inflammatory response induced by trastuzumab, as hypothesised by Koolen et al (2013).
In patients with HER2 + tumour, pCR is a powerful predictor of clinical outcome (Untch et al, 2011; Von Minckwitz et al, 2012). Early detection with interim PET of patients who are unlikely to achieve pCR should therefore be helpful to adapt treatment. Several recent works suggest that dual inhibition of HER2 (trastuzumab + lapatinib or trastuzumab + pertuzumab) has high efficacy (Baselga et al, 2012; Gianni et al, 2012; Guarneri et al, 2012). Another approach used bevacizumab together with trastuzumab and chemotherapy (Pierga et al, 2012). However, these strategies might also involve higher side effects; hence, the importance of patient selection. For example, although the overall pCR rates were increased by adding lapatinib to trastuzumab $(51.3 \%$ instead of $29.5 \% ; P=0.0001$ ), the frequencies of grade 3 diarrhoea, liver enzyme alterations, neutropenia and skin disorders were higher (Baselga et al, 2012). Better tolerance was reported when adding 

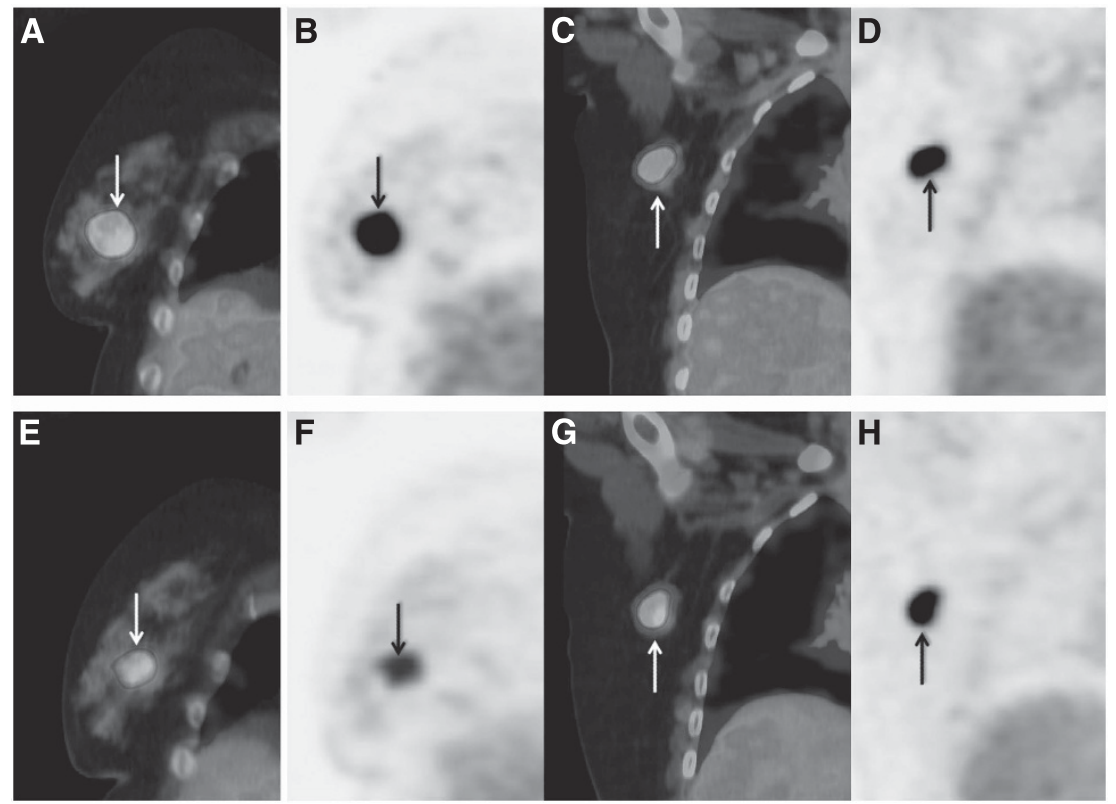

Figure 4. A 49-year-old woman (patient no. 20; Table 2) with HER2-overexpressing tumour of right breast. Positron emission tomography and PET/CT fusion images of the primary tumour ( $\mathbf{A}$ and $\mathbf{B}$ ) and an axillary lymph node (C and $\mathbf{D})$ at baseline and corresponding images after two courses of chemotherapy (E-H). At baseline SUV max was 11 in breast tumour (arrow, $\mathbf{A}$ and $\mathbf{B}$ ) and 16.2 in axillary lymph node (target lesion) (arrow, C and D). At $\mathrm{PET}_{2}, \mathrm{SUV}_{\max }$ was 5.9 in breast tumour (arrow, $\mathrm{E}$ and $\mathbf{F}$ ) $(\Delta \mathrm{SUV}=-46 \%$ ), and 13.4 in the lymph node (arrow, $\mathbf{G}$ and $\mathbf{H}$ ) $(\Delta \mathrm{SUV}=-17 \%)$. At completion of NAT, surgery showed residual invasive tumour.

pertuzumab to trastuzumab (Gianni et al, 2012). When poor responders are to be selected for novel treatment strategies within clinical trials, the role of early metabolic response prediction is important.

Because data from the present prospective study were acquired in a single institution, the criteria for metabolic response that we raise need validation in large multicentric studies.

\section{CONCLUSION}

In patients with HER2 + breast cancer, interim 18F-FDG-PET/CT after two cycles of chemotherapy allowed early identification of poor metabolic responders who are at high risk of residual tumour after completion of NAT with chemotherapy + trastuzumab. Because alternative strategies are now available (e.g., chemotherapy + dual anti-HER2 blockade, addition of an antiangiogenic), early prediction can be helpful for patient selection within clinical trials.

\section{CONFLICT OF INTEREST}

The authors declare no conflict of interest.

\section{REFERENCES}

Arnould L, Gelly M, Penault-Llorca F, Benoit L, Bonnetain F, Migeon C, Cabaret V, Fermeaux V, Bertheau P, Garnier J, Jeannin JF, Coudert B (2006) Trastuzumab-based treatment of HER2-positive breast cancer: an antibody-dependent cellular cytotoxicity mechanism? Br J Cancer 94: 259-267.

Baselga J, Bradbury I, Eidtmann H, Di Cosimo S, de Azambuja E, Aura C, Gómez H, Dinh P, Fauria K, Van Dooren V, Aktan G, Goldhirsch A, Chang TW, Horváth Z, Coccia-Portugal M, Domont J, Tseng LM, Kunz G, Sohn JH, Semiglazov V, Lerzo G, Palacova M, Probachai V, Pusztai L, Untch M, Gelber RD, Piccart-Gebhart M. NeoALTTO Study Team (2012) Lapatinib with trastuzumab for HER2-positive early breast cancer (NeoALTTO): a randomised, open-label, multicentre, phase 3 trial. Lancet 379: 633-640.

Boellaard R, O'Doherty MJ, Weber WA, Mottaghy FM, Lonsdale MN, Stroobants SG, Oyen WJ, Kotzerke J, Hoekstra OS, Pruim J, Marsden PK, Tatsch K, Hoekstra CJ, Visser EP, Arends B, Verzijlbergen FJ, Zijlstra JM, Comans EF, Lammertsma AA, Paans AM, Willemsen AT, Beyer T, Bockisch A, Schaefer-Prokop C, Delbeke D, Baum RP, Chiti A, Krause BJ (2010) FDG PET and PET/CT: EANM procedure guidelines for tumour PET imaging: version 1.0. Eur J Nucl Med Mol Imag 37: 181-200.

Cochet A, Kerrou K, Nabholtz JM, Cachin F, Pierga JY, Champion L, Ferrero JM, Darcourt J, Petit T, Bourahla K, Bougnoux P, Baulieu JL, Dupre PF, Salaun PY, Bachelot TD, Mognetti T, Coeffic DE, Mesnard N, Coudert B, Berriolo-Riedinger A (2012) An open-label randomized, multicenter, phase II study on neoadjuvant treatment with trastuzumab plus docetaxel versus trastuzumab plus docetaxel plus bevacizumab according to positron emission tomography (PET) value modification in patients with early stage HER2-positive breast cancer (AVATAXHER): Design description. $J$ Clin Oncol 30: (Suppl; abstr TPS646).

Fuster D, Duch J, Paredes P, Velasco M, Muñoz M, Santamaría G, Fontanillas M, Pons F (2008) Preoperative staging of large primary breast cancer with $[18 \mathrm{~F}]$ fluorodeoxyglucose positron emission tomography/computed tomography compared with conventional imaging procedures. J Clin Oncol 26: 4746-4751.

Gebhart G, Flamen P, Robles Barba J, Holmes E, Garcia C, Cortes Romera M, Eidtmann H, Baselga J, Gamez Cenzano C (2012) FDG-PET/CT for early prediction of response to neoadjuvant anti-HER2 therapies in HER2-positive breast cancer (BC) patients. J Nucl Med 53: 60 (Suppl; Abstracts).

Gianni L, Eiermann W, Semiglazov V, Manikhas A, Lluch A, Tjulandin S, Zambetti M, Vazquez F, Byakhow M, Lichinitser M, Climent MA, Ciruelos E, Ojeda B, Mansutti M, Bozhok A, Baronio R, Feyereislova A, Barton C, Valagussa P, Baselga J (2010) Neoadjuvant chemotherapy with trastuzumab followed by adjuvant trastuzumab versus neoadjuvant chemotherapy alone, in patients with HER2-positive locally advanced breast cancer (the NOAH trial): a randomised controlled superiority trial with a parallel HER2-negative cohort. Lancet 375: 377-384.

Gianni L, Pienkowski T, Im YH, Roman L, Tseng LM, Liu MC, Lluch A, Staroslawska E, de la Haba-Rodriguez J, Im SA, Pedrini JL, Poirier B, Morandi P, Semiglazov V, Srimuninnimit V, Bianchi G, Szado T, Ratnayake J, Ross G, Valagussa P (2012) Efficacy and safety of neoadjuvant pertuzumab and trastuzumab in women with locally advanced, inflammatory, or early HER2-positive breast cancer 
(NeoSphere): a randomised multicentre, open-label, phase 2 trial. Lancet Oncol 13: 25-32.

Groheux D, Giacchetti S, Delord M, Hindié E, Vercellino L, Cuvier C, Toubert ME, Merlet P, Hennequin C, Espié M (2013a) ${ }^{18}$ F-FDG PET/CT in staging patients with locally advanced or inflammatory breast cancer: comparison to conventional staging. J Nucl Med 54: 5-11.

Groheux D, Giacchetti S, Espié M, Rubello D, Moretti JL, Hindié E (2011a) Early monitoring of response to neoadjuvant chemotherapy in breast cancer with (18)F-FDG PET/CT: defining a clinical aim. Eur J Nucl Med Mol Imag 38: 419-425.

Groheux D, Giacchetti S, Moretti JL, Porcher R, Espié M, Lehmann-Che J, de Roquancourt A, Hamy AS, Cuvier C, Vercellino L, Hindié E (2011b) Correlation of high (18)F-FDG uptake to clinical, pathological and biological prognostic factors in breast cancer. Eur J Nucl Med Mol Imag 38: 426-435.

Groheux D, Hatt M, Hindié E, Giacchetti S, De Cremoux P, Lehmann-Che J, Martineau A, Marty M, Cuvier C, Cheze-Le Rest C, de Roquancourt A, Visvikis D, Espié M (2013b) Estrogen receptor-positive/human epidermal growth factor receptor 2-negative breast tumors: early prediction of chemosensitivity with (18) F-fluorodeoxyglucose positron emission tomography/computed tomography during neoadjuvant chemotherapy. Cancer 119: 1960-1968.

Groheux D, Hindié E, Delord M, Giacchetti S, Hamy AS, de Bazelaire C, de Roquancourt A, Vercellino L, Toubert ME, Merlet P, Espié M (2012a) Prognostic impact of ${ }^{18} \mathrm{FDG}$-PET-CT findings in clinical stage III and IIB breast cancer. J Natl Cancer Inst 104: 1879-1887.

Groheux D, Hindié E, Giacchetti S, Delord M, Hamy AS, de Roquancourt A, Vercellino L, Berenger N, Marty M, Espié M (2012b) Triple-negative breast cancer: early assessment with ${ }^{18} \mathrm{~F}$-FDG PET/CT during neoadjuvant chemotherapy identifies patients who are unlikely to achieve a pathologic complete response and are at a high risk of early relapse. J Nucl Med $\mathbf{5 3}$ : 249-254.

Guarneri V, Frassoldati A, Bottini A, Cagossi K, Bisagni G, Sarti S, Ravaioli A, Cavanna L, Giardina G, Musolino A, Untch M, Orlando L, Artioli F, Boni C, Generali DG, Serra P, Bagnalasta M, Marini L, Piacentini F, D'Amico R, Conte P (2012) Preoperative chemotherapy plus trastuzumab lapatinib, or both in human epidermal growth factor receptor 2-positive operable breast cancer: results of the randomized phase II CHER-LOB Study. J Clin Oncol 30: 1989-1995.

Hatt M, Groheux D, Martineau A, Espié M, Hindié E, Giacchetti S, de Roquancourt A, Visvikis D, Cheze-Le Rest C (2013) Comparison between ${ }^{18}$ F-FDG PET image-derived indices for early prediction of response to neoadjuvant chemotherapy in breast cancer. J Nucl Med 54: 341-349.

Humbert O, Berriolo-Riedinger A, Riedinger JM, Coudert B, Arnould L, Cochet A, Loustalot C, Fumoleau P, Brunotte F (2012) Changes in ${ }^{18}$ F-FDG tumor metabolism after a first course of neoadjuvant chemotherapy in breast cancer: influence of tumor subtypes. Ann Oncol 23: 2572-2577.

Koolen BB, Pengel KE, Wesseling J, Vogel WV, Vrancken Peeters MJ, Vincent AD, Gilhuijs KG, Rodenhuis S, Rutgers EJ, Valdés Olmos RA (2013) FDG PET/CT during neoadjuvant chemotherapy may predict response in ER-positive/HER2-negative and triple negative, but not in HER2-positive breast cancer. Breast S0960-9776(13): 00002-00007.

NCCN Clinical Practice Guidelines in Oncology (2013) Breast Cancer. Version 3 (2013). Available at http://www.nccn.org/professionals/ physician_gls/f_guidelines.asp.
Pierga JY, Petit T, Delozier T, Ferrero JM, Campone M, Gligorov J, Lerebours F, Roché H, Bachelot T, Charafe-Jauffret E, Pavlyuk M, Kraemer S, Bidard FC, Viens P (2012) Neoadjuvant bevacizumab, trastuzumab, and chemotherapy for primary inflammatory HER2-positive breast cancer (BEVERLY-2): an open-label, single-arm phase 2 study. Lancet Oncol 13: 375-384.

Rousseau C, Devillers A, Campone M, Campion L, Ferrer L, Sagan C, Ricaud M, Bridji B, Kraeber-Bodéré F (2011) FDG PET evaluation of early axillary lymph node response to neoadjuvant chemotherapy in stage II and III breast cancer patients. Eur J Nucl Med Mol Imag 38: 1029-1036.

Schwarz-Dose J, Untch M, Tiling R, Sassen S, Mahner S, Kahlert S, Harbeck N, Lebeau A, Brenner W, Schwaiger M, Jaenicke F, Avril N (2009) Monitoring primary systemic therapy of large and locally advanced breast cancer by using sequential positron emission tomography imaging with $\left[{ }^{18} \mathrm{~F}\right]$ fluorodeoxyglucose. J Clin Oncol 27: 535-541.

Smith TA, Appleyard MV, Sharp S, Fleming IN, Murray K, Thompson AM (2013) Response to trastuzumab by HER2 expressing breast tumour xenografts is accompanied by decreased hexokinase II, glutl and [(18)F]-FDG incorporation and changes in (31)P-NMR-detectable phosphomonoesters. Cancer Chemother Pharmacol 71: 473-480.

Straver ME, Aukema TS, Olmos RA, Rutgers EJ, Gilhuijs KG, Schot ME, Vogel WV, Peeters MJ (2010) Feasibility of FDG PET/CT to monitor the response of axillary lymph node metastases to neoadjuvant chemotherapy in breast cancer patients. Eur J Nucl Med Mol Imag 37: 1069-1076.

Untch M, Fasching PA, Konecny GE, Hasmüller S, Lebeau A, Kreienberg R, Camara O, Müller V, du Bois A, Kühn T, Stickeler E, Harbeck N, Höss C, Kahlert S, Beck T, Fett W, Mehta KM, von Minckwitz G, Loibl S (2011) Pathologic complete response after neoadjuvant chemotherapy plus trastuzumab predicts favorable survival in human epidermal growth factor receptor 2-overexpressing breast cancer: results from the TECHNO trial of the AGO and GBG study groups. J Clin Oncol 29: 3351-3357.

Von Minckwitz G, Untch M, Blohmer JU, Costa SD, Eidtmann H, Fasching PA, Gerber B, Eiermann W, Hilfrich J, Huober J, Jackisch C, Kaufmann M, Konecny GE, Denkert C, Nekljudova V, Mehta K, Loibl S (2012) Definition and impact of pathologic complete response on prognosis after neoadjuvant chemotherapy in various intrinsic breast cancer subtypes. J Clin Oncol 30: 1796-1804.

Wang Y, Zhang C, Liu J, Huang G (2012) Is ${ }^{18}$ F-FDG PET accurate to predict neoadjuvant therapy response in breast cancer? A meta-analysis. Breast Cancer Res Treat 131: 357-369.

Wolff AC, Hammond ME, Schwartz JN, Hagerty KL, Allred DC, Cote RJ, Dowsett M, Fitzgibbons PL, Hanna WM, Langer A, McShane LM, Paik S, Pegram MD, Perez EA, Press MF, Rhodes A, Sturgeon C, Taube SE, Tubbs R, Vance GH, van de Vijver M, Wheeler TM, Hayes DF. American Society of Clinical Oncology; College of American Pathologists (2007) American Society of Clinical Oncology/College of American Pathologists guideline recommendations for human epidermal growth factor receptor 2 testing in breast cancer. J Clin Oncol 25: 118-145.

This work is published under the standard license to publish agreement. After 12 months the work will become freely available and the license terms will switch to a Creative Commons AttributionNonCommercial-Share Alike 3.0 Unported License. 